\title{
Estudo retrospectivo de radiografias com fraturas rádio e ulna em cães
}

Robson Fortes GIGLIO ${ }^{1}$

Franklin de Almeida

STERMAN ${ }^{1}$

Ana Carolina Brandão de Campos FONSECA PINTO ${ }^{1}$

Silvana Maria UNRUH ${ }^{1}$

Alexandre SCHMAEDECKE ${ }^{1}$

Cassio Ricardo Auada

FERRIGNO $^{1}$

\section{Correspondência para:}

Av. Marte, 125. apto 33, Alphaville, Santana de Parnaíba, São Paulo, 06483-310 e-mail: rgiglio@usp.br

Recebido para publicação: 08/06/2006 Aprovado para publicação: 23/08/2007

\begin{abstract}
1 - Departamento de Cirurgia da Faculdade de Medicina Veterinária e Zootecnia da Universidade de São Paulo, São Paulo-SP
\end{abstract}

\section{Resumo}

Palavras-chave: Radiografia.

Foram analisadas 528 radiografias simples de fraturas de rádio e ulna do arquivo do Serviço de Diagnostico por Imagem do Departamento de Cirurgia junto ao Hospital Veterinário da Faculdade de Medicina Veterinária e Zootecnia da Universidade de São Paulo realizadas no período de julho de 1999 a dezembro de 2005, selecionando-se os meses para análise de forma randomizada. Os principais resultados encontrados foram: Fraturas de rádio e ulna representam 20,9\% do total encontradas, destas o tipo mais observado foi a transversal, tanto no rádio $(75,5 \%)$ quanto na ulna $(76,6 \%) .87,1 \%$ das fraturas acometeram o rádio e a ulna. $\mathrm{O}$ terço distal foi o mais acometido foi o distal, tanto do rádio quanto da ulna $(72,9 \%$ e $70,1 \%$, respectivamente). Esquírolas ósseas foram observadas em 13,8\% e desvio de eixo ósseo em $94,9 \%$ dos casos. 40,2\% dos cães não possuíam raça definida. $34,3 \%$ dos cães possuíam idade entre seis meses a um ano.

\section{Introdução}

Fraturas de vários tipos podem ser observadas nos ossos rádio e ulna, afetando-os de forma individual ou conjunta. ${ }^{1}$ Entretanto, as diafisárias, em ambos os ossos, resultantes de traumas são observadas com maior freqüência., ${ }^{2,3}$ Geralmente as fraturas nos terços médio e distal destes ossos são observadas da forma conjunta, como uma unidade. ${ }^{4}$ A incidência de complicações como má e não-uniões é relativamente alta, principalmente em raças de pequeno porte. ${ }^{1}$ No estudo feito por Vaughan ${ }^{5}$, as fraturas de rádio e ulna constituíram 60\% do total de não-uniões observadas. As fraturas de extremidade distal, como a dos processos estilóides da ulna, podem indicar instabilidade articular com o carpo. ${ }^{6}$ As fraturas de rádio e ulna representam cerca de $8,5 \%$ a $18 \%$ do total das fraturas nos estudos de diversos serviços e autores. ${ }^{1,3,7,8,9,10}$

\section{Material e Método}

No período de julho de 1999 a dezembro de 2005 selecionou-se, de forma randomizada, casos de 20 meses. Em cada mês selecionado, pesquisou-se nos arquivos de laudos do Serviço de Diagnostico por Imagem do Departamento de Cirurgia junto ao Hospital Veterinário da Faculdade de Medicina Veterinária e Zootecnia da Universidade de São Paulo todas as fraturas agudas (sem sinais de cronicidade, como bordas arredondadas, reação periostal, osteoesclerose etc.) radiografadas nos meses selecionados. A partir daí, estudou-se as fraturas agudas de rádio e ulna do arquivo de radiografias do Serviço supracitado, excluindo-se fraturas secundárias a alterações metabólicas ou neoplasias ósseas.

As radiografias foram classificadas de acordo com os seguintes critérios de análise: total de fraturas de rádio e ulna no total de fraturas em cães, tipo de fratura, membro acometido, osso acometido, localização da fratura, presença ou não de esquírolas, 
presença ou não de desvio ósseo, raça, idade e sexo. Os resultados obtidos foram expressos mediante a distribuição de freqüências e respectivas porcentagens.

\section{Resultados e Discussão}

Do total de 2.526 fraturas agudas observadas no período de estudo, 528 foram de rádio e ulna, representando $20,9 \%$ do total, sendo maior que a encontrada por outros autores $1,3,7,8,9,10$, cujos valores situamse entre $8,5 \%$ a $18 \%$.

Em relação às raças, verificou-se que $213(40,2 \%)$ dos cães não possuíam raça definida, 117 (22,2\%) eram da raça Pinscher, $107(20,3 \%)$ Poodle, 17 (3,2\%) Terrier Brasileiro, 15 (2,9\%) Pastor Alemão, nove $(1,7 \%)$ American Pit Bull Terrier e 50 (9,5\%) perfazem o total de outras raças. O maior número de fraturas em cães sem raça definida pode ser explicado porque estes cães representam a maioria dos que são atendidos ao Hospital Veterinário da Universidade de São Paulo, o que dificulta, de outra parte, a avaliação por porte dos animais devido a grandes diferenças constitucionais entre os animais desse grupo. No entanto, os cães das três colocações seguintes $\left(2^{\circ}, 3^{\circ}\right.$ e $4^{\circ}$ posições $)$, que representam $45,7 \%$ do total, são cães de pequeno porte, demonstrando maior fragilidade destes a traumas no antebraço.

No atinente ao sexo, observou-se maior número de casos em fêmeas $(53,2 \%)$ do que em machos $(46,8 \%)$. O resultado vem de encontro ao estudo de Phillips ${ }^{9}$, que realizando levantamento geral de fraturas em cães, observou maior percentagem de macho $(68,3 \%)$ que de fêmeas $(31,7 \%)$.

No que diz respeito à idade, verificouse que $119(22,6 \%)$ cães tinham até seis meses, $181(34,3 \%)$ entre seis meses e um ano, 68 $(12,8 \%)$ de um até dois anos, $38(7,2 \%)$ entre dois e três anos, três animais $(0,6 \%)$ de três a quatro anos, $24(4,6 \%)$ entre quatro e cinco anos, $77(14,5 \%)$ acima de cinco anos e 18 $(3,4 \%)$ cães com idade desconhecida. Estes dados estão de acordo com Huise e Johnson ${ }^{2}$, que relatam predisposição dos animais jovens, visto que, no presente estudo $56,9 \%$ dos cães possuíam até um ano de idade. No entanto, os dados deste estudo diferem dos apresentados por Phillips ${ }^{9}$, no qual a idade mais acometida foi até seis meses, com $28,6 \%$ dos casos do seu levantamento sobre fraturas.

O membro torácico direito, com 246 $(46,6 \%)$ casos, foi discretamente mais acometido que o esquerdo, que somou 232 $(43,9 \%)$ dos casos. O acometimento bilateral foi observado em $25(9,5 \%)$ dos casos.

Em $87,1 \%$ das fraturas, rádio e ulna foram acometidos ambos e, também, foram observadas fraturas somente no rádio em $2,7 \%$ e, somente na ulna, em $10,2 \%$ do total de casos. Valores diferentes foram observados por Phillips ${ }^{9}$, que estudou 49 casos de fraturas de rádio e ulna nos quais em 34 casos $(69,4 \%)$ houve fraturas nos dois ossos, $9(18,4 \%)$ somente na ulna e $6(12,2 \%)$ somente no rádio.

Em relação ao tipo de fratura no rádio, foram observadas fraturas transversas em $358(75,5 \%)$ casos, fraturas oblíquas em $110(23,2 \%)$ casos e $6(1,3 \%)$ de fraturas cominutivas. $\mathrm{Na}$ ulna foram observadas fraturas transversas em $394(76,6 \%)$ casos, fraturas oblíquas em $112(21,8 \%)$ casos e oito $(2,0 \%)$ de fraturas cominutivas.

No rádio, constatou-se $36(6,5 \%)$ de fraturas no terço proximal, $114(20,6 \%)$ no terço médio e $404(72,9 \%)$ no terço distal. Na ulna, observou-se $64(10,7 \%)$ fraturas no terço proximal, $114(19,2 \%)$ no terço médio e $417(70,1 \%)$ no terço distal. De acordo com Rudd e Whitehair ${ }^{1}$ e Lappin et al. ${ }^{3}$ as fraturas acometem com maior freqüência o terço médio e distal de rádio e ulna. No estudo de Lappin et al. ${ }^{3}$ que estudaram 105 fraturas, $45(42,8 \%)$ foram observadas no terço distal, $51(48,5 \%)$ no terço médio e 9 $(8,6 \%)$ no terço distal. No presente estudo, o terço distal foi o mais acometido de rádio e ulna, seguido pelo terço médio e terço proximal. A observação de apenas 36 (6,5\%) de casos de fratura no terço proximal de rádio confirma as informações de Huise e Johnson ${ }^{2}$, que asseguram que é raro ocorrer fratura nesse terço do rádio. Das fraturas de 
ulna estudadas, 21 ocorreram no olécrano, representando $3,5 \%$ de todas as fraturas de ulna e $32,8 \%$ das fraturas do seu terço proximal, valores destoantes aos encontrados por Phillips ", que observou cinco fraturas de olécrano em nove fraturas de terço proximal de ulna. Também foram observadas 23 fraturas de processo estilóide da ulna, representando 3,9\% das fraturas de ulna e $5,5 \%$ das fraturas de seu terço distal.

Quanto ao desvio de eixo ósseo, observou-se que $501(94,9 \%)$ fraturas apresentaram desvio, sendo que em 126 $(23,8 \%)$ este desvio foi considerado discreto e em $40(7,6 \%)$ casos foi considerado acentuado.

No atinente a presença de esquírolas, observou-se que em $73(13,8 \%)$ casos observou-se a presença de pelo menos uma esquírola óssea.

A importância deste estudo retrospectivo está no número grande de radiografias analisadas (528 radiografias de fraturas de rádio e ulna), quantidade não observada em nenhum estudo do gênero até o presente momento, possibilitando maior confiabilidade dos resultados obtidos. Conhecendo os tipos e variações mais comuns das fraturas nestes ossos, os estudiosos e práticos da área de ortopedia, traumatologia e fisioterapia veterinária podem direcionar sua atenção para formas de correção, estabilização e aperfeiçoamento de técnicas nestes tipos de fraturas, aumento a eficiência no tratamento e reabilitação dos mesmos.

\title{
Retrospective study of radiographies with radius and ulna fractures in dogs
}

\begin{abstract}
Survey of 528 radiographs of radius and ulna fractures selected from archive of Radiology Service of the Surgery Department into the Veterinary Hospital of Veterinary College of the University of Sao Paulo from July 1999 - December 2005 we randomly selected the months to analyze. Radius and ulna fractures represent $20.9 \%$ of total fractures. $87.1 \%$ of these fractures attacked both bones. Transversal fractures were the most common type even in radius $(75.5 \%)$ than ulna $(76.6 \%)$, which occurred mainly at distal third radius $(72.9 \%)$ and ulna $(70.1 \%)$. Mix-breed dogs $(40.2 \%)$, between the ages of six months and one year $(34.3 \%)$, with displacement of axis bone $(94.9 \%)$ were the most frequent types observed.
\end{abstract}

\section{Referências}

1 RUDD, R. G.; WHITEHAIR, J. G. Fractures of the radius and ulna. Veterinary Clinics of North América: Small Animal Practice, v. 22, n. 1, p. 135-148, 1992.

2 HUISE, D. A; JOHNSON, A. L. Managment of specifics fractures. In: FOSSUM, T. W. Small animal surgery. $1^{\text {a }}$ ed. St. Louis: Mosby, 1997. p. 803-818.

3 LAPPIN, M. R. et al. Fractures of the radius and ulna in the dog. Journal of the American Animal Hospital Association, n. 19, p. 643-650, 1983.

4 BRINKER, W. O.; PIERMATTEI, D. L.; FLO, G. L.: Fraturas de rádio e ulna. In: BRINKER, W. O.; PIERMATTEI, D. L.; FLO, G. L, Manual de ortopedia dos pequenos animais. $3^{\text {a }}$ ed. São Paulo: Manole, 1983. p. 301.

5 VAUGHAN, L. C. A Clinical study of nonunion fractures in the dog. Journal of Small Animal Practice, v. 5, n. 8, p. 173-177, 1964.
Key words:

Radiography.

Dogs.

Fracture.

Radius.

Ulna.
6 JOHNSON, K. A. Carpal injuries. In.: BLOOMBERG, M. S.; DEE, J. F.; TAYLOR, R. A. Canine sports medicine and surgery. Philadelphia: WB Saunders, 1998. p. 100119.

7 LEONARD, E. P. Radius and ulna. In: LEONARD, E. $P$. Orthopedic surgery in the dog and cat. $2^{a}$ ed. Philadelphia: WB Saunders, 1971. p. 149.

8 ORMROD, A. N. Symposium on limb fractures in the dog and cat: IV Fractures of forelimb. Journal of Small Animal Practice, n. 7, p. 155-162, 1966.

9 PHILLIPS, I. R. A Survey of bone fractures in the dog and cat. Journal of Small Animal Practice, v. 20, n. 11, p. 661-674, 1979.

10 SUMNER-SMITH, G.; CAWLEY, A .J. Nonunion of fractures in the dog. Journal of Small Animal Practice, n. 11 , p. $311-325,1970$.

11 SUMNER-SMITH G. Bone plating for radial fractures in small dog. Model Veterinary Practice, n. 3, p. 30-33, 1970. 